\title{
Identificação da espiral de Fibonacci no padrão de crescimento vegetal por meio de modelo 3D virtual obtido através de fotogrametria digital
}

Identification of the Fibonacci spiral in the plant growth pattern using a virtual $3 D$ model obtained through digital photogrammetry

ANDRADE, Rebeca M.; Mestranda; PGDesign UFRGS

rebecadeandrade@gmail.com

DETANICO, Flora B.; Doutoranda; PGDesign UFRGS

floradetanico@me.com

SILVA, Fábio P.; Doutor; PGDesign UFRGS

fabio.silva@ufrgs.br

TEIXEIRA, Fábio G.; Doutor; PGDesign UFRGS

fabiogt@ufrgs.br

\section{Resumo}

A investigação e análise dos padrões formais e estruturais da natureza fornecem dados de considerável potencial resolutivo para auxílio na solução de problemas humanos de ordem técnica, funcional e estética. $O$ objetivo deste trabalho é identificar relações geométricas tais como a proporção áurea e a sequência de Fibonacci nos padrões de crescimento vegetal. Para realização da pesquisa foi selecionada uma espécie de suculenta, popularmente conhecida como Flor Fantasma (nome científico: Graptopetalum paraguayense), a qual passou por um processo de Fotogrametria Digital, uma das técnicas de digitalização tridimensional, utilizada para a obtenção de um modelo 3D virtual. O modelo 3D foi submetido à análise e manipulação digital, possibilitando identificar, no objeto de estudo, uma geometria com geratrizes helicoidais similares às encontradas na Espiral de Fibonacci.

Palavras Chave: Espiral de Fibonacci, Padrão de Crescimento Vegetal, Biônica, Biomimética, Digitalização 3D, Fotogrametria

\section{Abstract}

The research and analysis of the regular and structural patterns from nature provides data of considerable resolving potential to solution of technical, functional and aesthetic human problems. This work aims to identify geometric relations such as the golden ratio and the Fibonacci sequence in plant growth patterns. To conduct the research was selected one kind of succulent plant, popularly known as Ghost Plant (botanical name: Graptopetalum paraguayense), which was submitted to a process of Digital Photogrammetry, which is a kind of three-dimensional scanning techniques which can be used to obtain virtual 3D models. The obtained 3D model was submitted 
to digital analysis and manipulation, making it possible to identify, in the object of study, a geometry which has helical generatrix similar to those ones found in the Fibonacci Spiral

Keywords: Fibonacci Spiral, Plant Growth Pattern, Bionics, Biomimetics, 3D Scanning, Photogrammetry

\section{Introdução}

A análise dos padrões formais da natureza oferece grande potencial resolutivo para o campo do design. As espécies vegetais, por exemplo, apresentam impressionantes modelos de organização espacial, os quais podem ser investigados e transpostos ao design através de parâmetros geométricos. São belos exemplos desses padrões geométricos espontâneos as sementes de girassol e a estrutura dos gomos da pinha, os quais apresentam duplas espirais regradas pela proporção áurea e pela sequência de Fibonacci (HAUDIN e RESIDORI, 2013).

Segundo o pesquisador Hsuan-an (2002), que estuda diferentes tipos de sementes do cerrado brasileiro, dentro da aleatoriedade aparente apresentada pelas formas naturais, está oculta certa ordem, caracterizando uma aparência livre e espontânea, porém não aleatória, visto que sempre há nelas algum padrão organizacional. O mesmo sugere Doczi (1990) nos seus gráficos que apresentam geometricamente diferentes formas da natureza (vegetais, animais e humanas) indicando semelhanças e padrões de repetição. No estudo da margarida, por exemplo, o autor identifica espirais sinérgicas como geratrizes criadoras do crescimento orgânico.

A observação da existência de uma coincidência formal entre o padrão de crescimento vegetal e alguns padrões geométricos, aponta para um vasto campo de investigação, estudo e exploração em diferentes áreas. Por meio de técnicas de engenharia reversa é possível chegar às geratrizes organizacionais a partir de um modelo já constituído. O presente trabalho apresenta o estudo e análise de um modelo tridimensional (3D) virtual da suculenta Flor Fantasma (nome científico: Graptopetalum paraguayense), obtido por meio de Fotogrametria Digital, que é uma técnica de digitalização tridimensional.

Conforme definições da Britannica Digital Learning ("Photogrammetry", 2017), Fotogrametria é uma técnica que surgiu com a finalidade de, através de fotografias, fazer mapeamento e levantamento cartográfico. Embora a Fotogrametria aérea tenha sido muito utilizada para fins militares até o final da Segunda Guerra Mundial, na atualidade é o principal método de elaboração de mapas, especialmente de áreas de difícil acesso, sendo também muito utilizada em estudos relacionados à Ecologia e também em estudos de outras áreas. Originalmente a Fotogrametria pertence ao campo do Sensoriamento Remoto, sendo uma tradicional área de estudo e prática da Geodésia (LINDER, 2009).

A escolha da Fotogrametria Digital como técnica de digitalização decorreu das limitações encontradas no objeto de estudo. Com base nas características dimensionais, formais e estruturais, percebeu-se que o processo de digitalização mais adequado seria a Fotogrametria, já que o objeto de estudo fica estático sobre uma base que gira gradualmente enquanto fotografias são tiradas de diferentes posições (ângulos $x$ alturas).

Foram capturadas um total de 255 fotografias (sendo que 38 não foram utilizadas) que, 
após serem processadas por um software especializado, possibilitaram a obtenção do modelo 3D virtual. O modelo obtido foi então analisado em outro software, no qual foi possível identificar e marcar pontos, realizar medições e ainda fazer comparações entre as geratrizes helicoidais encontradas no modelo e as encontradas na espiral de Fibonacci.

\section{Fotogrametria digital}

A Fotogrametria Digital é uma tecnologia de informação que vem sendo usada na geração de informações geométricas, radiométricas e também de semântica a respeito de objetos tridimensionais (HEIPKE, 1995). Essa tecnologia está se tornando cada vez mais acessível devido, principalmente, ao grau de autonomia que se tem quanto a captura das imagens, que pode ser feita até com o uso da câmera de um telefone celular, e também em decorrência do constante desenvolvimento no campo computacional, que possibilita um processo menos analógico, já que toda a parte de cálculos é feita a armazenada no computador (Linder, 2009). Os conhecimentos em Fotogrametria apresentam um crescimento surpreendente, seja no âmbito da arte ou no da ciência, sendo que vem se consolidando como um instrumento cada vez mais necessário no âmbito da cartografia no mundo (TEMBA, 2000).

Fotogrametria, como definido por Foster e Halbstein (2014), é a ciência pela qual, através de imagens bidimensionais (2D), geralmente fotografias, são extraídas medições confiáveis. Reforçando os conceitos apresentados por Foster e Halbstein (2014), estudos feitos pela Sociedade Americana de Fotogrametria e Sensoriamento Remoto ${ }^{1}$ apontam para o fato de que a Fotogrametria é a arte, ciência e tecnologia de obter informação verdadeiramente confiáveis de objetos físicos e seu entorno, por meio de procedimentos de gravação, medição e interpretação de fotografias. Seguindo a mesma linha de definição, Caro (2012) aponta que a Fotogrametria é uma técnica que permite fazer medições 3D e também obter volumes a partir de fotografias.

O uso de modelos 3D virtuais, conforme Arévalo Vera et al. (2015), têm permitido uma maior interatividade entre indivíduos e objetos, proporcionando uma relação que vai além da visualização direta, passando a uma visualização virtual, pois essa relação permite exploração e estudo de objetos e ambientes, como peças de patrimônios, mapas e construções, sem que haja alteração de formas e também sem que a perspectiva da visão humana sofra alterações. Ainda conforme apontamentos dos autores, o uso de modelos 3D virtuais têm permitido também a realização de observações e estudos em diversas áreas, pois o uso da Fotogrametria Digital é um recurso que possibilita a análise profunda de detalhes que não podem ser reconhecidos a olho nu.

Essa técnica vem sendo usada há mais de um século, no entanto foi a partir de 1990 que a fotogrametria digital apresentou um rápido desenvolvimento (SILVA, 2015), provavelmente ocasionado pela popularização das câmeras digitais e também o surgimento de softwares para processamento das fotografias. Atualmente existem disponíveis no mercado softwares e aplicativos projetados com foco em usuários não especializados, que em face de todo o processo de geração de modelos 3D virtuais, ficam responsáveis apenas pela etapa de captura das imagens que posteriormente serão processadas pelo software ou aplicativo (DANTAS et al., 2016). É possível encontrar softwares de uso livre e gratuito, e também opções com custo relativamente alto. Algumas dessas opções de alto custo costumam conceder licenças de uso gratuito a

\footnotetext{
${ }^{1}$ American Society for Photogrammetry and Remote Sensing - ASPRS (https://www.asprs.org).
} 
estudantes, como é o caso do ReCap $360^{\mathrm{TM}}$ da Autodesk, que foi utilizado para o processamento das imagens do presente trabalho.

A técnica para captura de fotos para Fotogrametria Digital requer o uso de alguns equipamentos e objetos que irão tornar o processo menos complexo e com maior chance de apresentar um resultado positivo. O processo, como um todo, pode ser dividido nos seguintes momentos: definição do tema (objeto ou cena) a ser fotografado; seleção dos objetos e equipamentos básicos; montagem do set e sessão fotográfica para captura das imagens e, por fim; submeter as fotografias ao processamento no software ou aplicativo que irá fazer a sobreposição que irá gerar o modelo 3D virtual.

A definição do tema (objeto ou cena) que será fotografado já irá indicar alguns requisitos referentes ao processo de digitalização tridimensional. Formato, tamanho, estrutura física, textura superficial, cores e detalhes são alguns dos elementos que irão influenciar diretamente na captura das imagens. Como visto em Sketchfab (2015), é recomendado evitar objetos que sejam completamente planos, muito finos, transparentes, brilhantes e/ou com superfície reflexiva. Folhas e plantas, pêlos, cabelo e utensílios de vidro são apenas alguns exemplos de objetos de difícil captura.

A relação de objetos e equipamentos básicos para Fotogrametria Digital, conforme visto em Sketchfab (2015), contempla: câmera fotográfica, computador, tripé, plataforma giratória e controle remoto (para o disparo da câmera). Destes, os que são fundamentais são a câmera e o computador, pois os outros podem ser improvisados e/ou adaptados com o uso de outros recursos. Outros elementos que podem ser usados são os marcadores (chamados targets), que são aplicados na superfície do modelo físico e também em seu entorno. Esses marcadores irão servir de auxílio ao software ou aplicativo no momento de junção das imagens, pois eles irão reconhecer as formas e calcular a melhor forma de fazer a sobreposição das fotografias.

\section{Metodologia}

Após a escolha do objeto de estudo, o desenvolvimento do trabalho se realizou em 4 etapas: 1) preparação do modelo físico, montagem do set e captura fotográfica, 2) processamento das fotografias no ReCap $360^{\mathrm{Tm}}$, 3) manipulação do modelo 3D virtual no Rhinoceros e início do processo de marcação de pontos e realização de medições, e 4) estabelecimento das similaridades entre geratrizes helicoidais encontradas no modelo virtual e as encontradas na espiral de Fibonacci. Cada uma das etapas será descrita de forma mais detalhada nos tópicos seguintes.

No que diz respeito à escolha de materiais, processos para digitalização e meio de manipulação do modelo 3D virtual, um dos requisitos técnicos para o desenvolvimento do trabalho foi considerar o uso de softwares gratuitos (considerando inclusive o uso de softwares pagos, mas que permitem o uso gratuito por meio de uma licença concedida a estudantes), objetos e equipamentos pessoais e também equipamentos e estruturas disponíveis em laboratórios da Faculdade de Engenharia e da Faculdade de Arquitetura, Urbanismo e Design da Universidade Federal do Rio Grande do Sul (UFRGS). 


\section{Artigo Completo}

\subsection{Preparação do modelo físico, montagem do set e sessão fotográfica}

Como abordado no item 2 do presente trabalho, alguns requisitos referentes ao processo de digitalização tridimensional já podem ser percebidos após a definição do tema (objeto ou cena) que será fotografado. As limitações encontradas no modelo selecionado para o trabalho indicavam que o processo mais adequado de digitalização seria a fotogrametria, que se justificava pelos dois fatores seguintes: o formato geral do modelo físico, que por possuir muitas folhas e em direções diferentes, causaria o efeito de sombra (também chamados de negativos), que é um problema, pois representa uma área que por não ser alcançada no momento da digitalização, ocasiona uma falha (um buraco) na malha gerada pelo software.

A estrutura física do modelo é o segundo fator, pois como ela não é rígida, o modelo pode se mover facilmente. Quando se trata de digitalização, o ideal é que o modelo permaneça estático durante todo o processo, para não gerar informação duplicada ou incorreta sobre o objeto. As cores e texturas superficiais não representaram nenhum tipo de limitação ao processo de digitalização, tampouco a forma das folhas, já que não eram pequenas e nem finas demais.

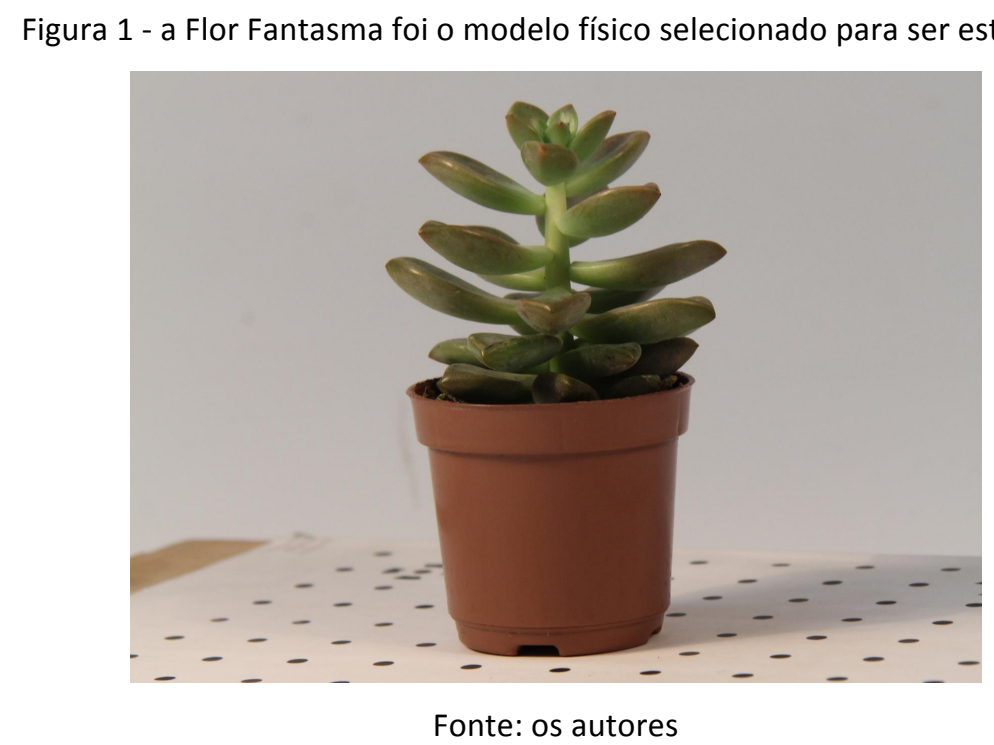

O processo de digitalização tridimensional por meio de Fotogrametria Digital pode ser feito com o uso de muita ou pouco tecnologia, com elevado ou baixo investimento. O presente trabalho demandou o uso de uma câmera digital Canon EOS 60D com lente de 18-200 mm, iluminação artificial projetada a partir de pontos diferentes e mesa giratória manual com targets. Todo o processo se deu de forma manual, sem o uso de tripé ou controle remoto para disparo da câmera. A rotação da mesa ocorreu também de forma manual, sem o uso de réguas. 


\section{Artigo Completo}

Figura 2 - montagem do set para sessão fotográfica: (a) mesa com fundo infinito e iluminação, e (b) modelo físico já posicionado sobre a superfície giratória que contém as targets.
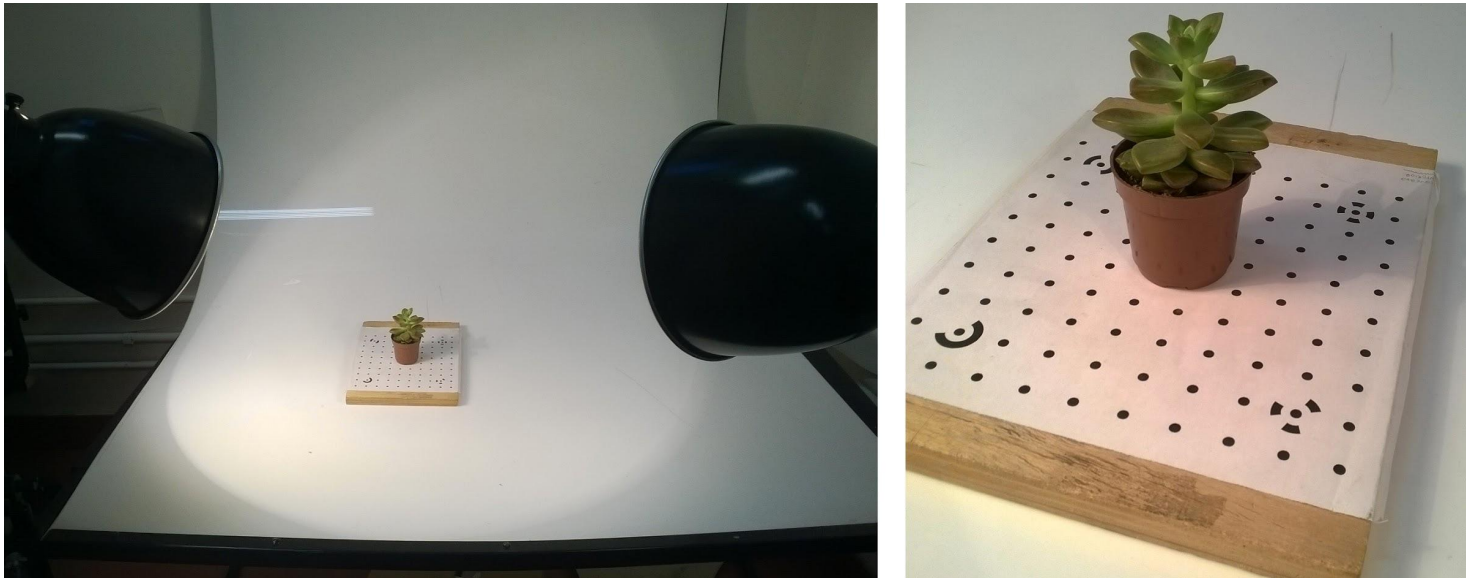

Fonte: os autores

O modelo foi fotografado de cinco diferentes posições (ângulos $\mathrm{x}$ alturas), e após cada uma dessas sequências de cinco capturas, a mesa foi rotacionada manualmente no sentido antihorário. 0 processo gerou um total de 255 fotografias, das quais apenas 217 foram utilizadas. As fotografias capturas foram então enviadas ao software citado na etapa 2 do item 3, para fazer o processamento e geração do modelo 3D virtual. No item 3.2 essas informações estão dispostas de forma mais detalhada.

\subsection{Processamento das fotografias}

Para o desenvolvimento dessa etapa, foi utilizado o software ReCap $360^{\mathrm{Tm}}$, por meio de uma licença educacional. Uma grande vantagem de se usar esse software é o fato de todo o processamento das imagens ser feito em um servidor remoto (o que chamamos de processamento na nuvem), então o usuário não necessita dispor de um computador de alto desempenho para gerar seus modelos 3D, precisando apenas estar conectado à internet.

Após o envio das imagens para o servidor do software, o usuário deve aguardar o recebimento de um e-mail informando que o modelo 3D virtual já está pronto e disponível em seu perfil pessoal. $\mathrm{O}$ modelo gerado pode ser exportado do software como arquivo na extensão .OBJ (Object File Wavefront 3D), que é um formato usado para objetos tridimensionais que contenham coordenadas 3D (pontos e linhas poligonais), mapas de textura e outras informações referentes ao objeto. Algumas vistas do modelo 3D virtual obtido podem ser vistas na Figura 3. 


\section{Artigo Completo}

Figura 3 - algumas vistas do modelo 3D obtido (janela de visualização do software).

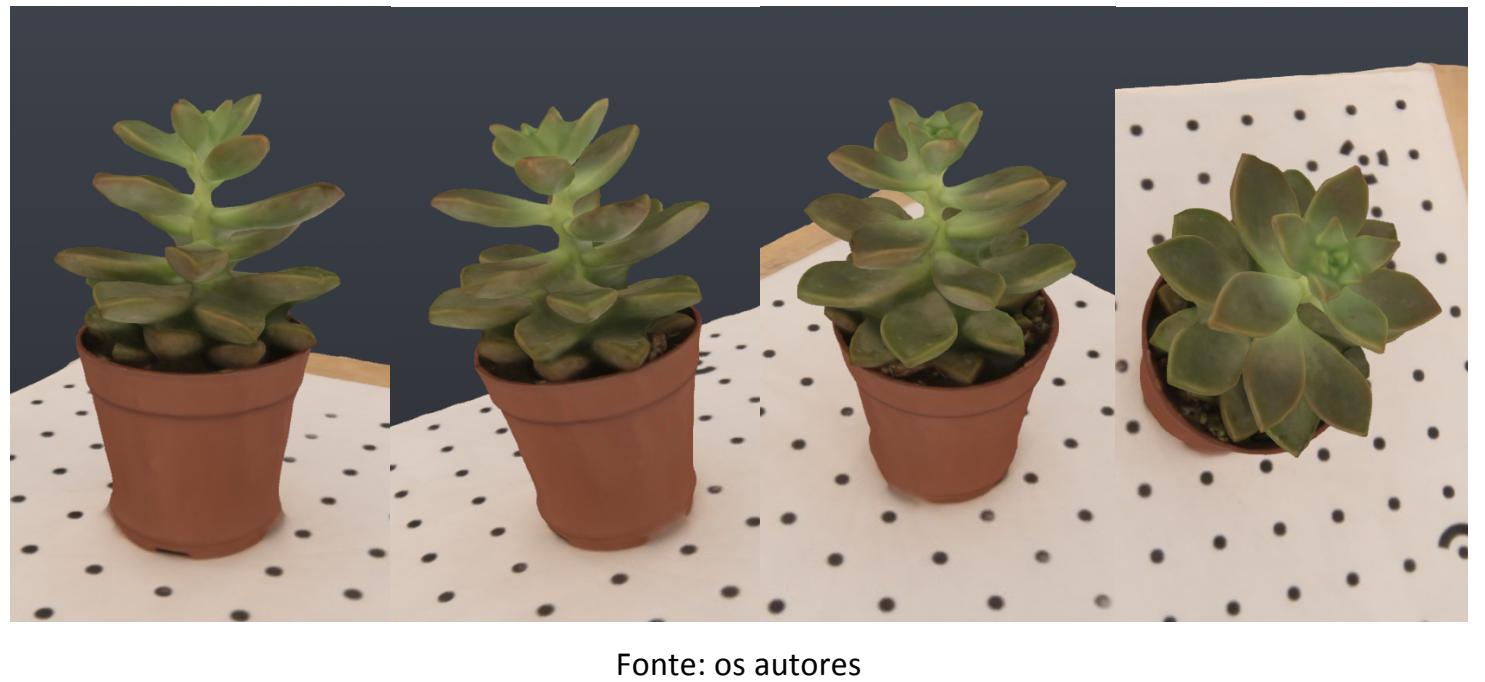

\subsection{Manipulação do modelo 3D virtual e início do processo de marcação de pontos e realização de medições}

O modelo 3D virtual obtido foi exportado para o Rhinoceros 3D com a extensão .OBJ, o que possibilitou sua manipulação e análise. A escolha por esse software se deu em função de sua diversidade de funcionalidades e comandos, excelente integração com outros programas, além da interface operacional ser intuitiva, com facilidade de visibilidade e ao mesmo tempo precisão dimensional. As vistas do modelo da suculenta ficaram bem delineadas e, a partir disso, foi possível traçar algumas geometrias para identificação da sua morfologia e padrões de crescimento.

A primeira tarefa executada foi a identificação e marcação de pontos no objeto. Situados nas extremidades das folhas da suculenta Flor Fantasma, esses pontos foram de fundamental importância no momento de medição do comprimento e da posição das folhas (a que distância se encontravam da linha de base). Para aferição das dimensões de comprimento das folhas, foi definido um eixo central, considerando o caule da planta e também o posicionamento do objeto num ângulo de 90ㅇ, possibilitando a extração da informação da cota de comprimento (Figura 4). 


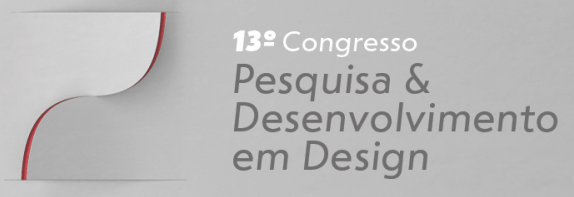

\section{Artigo Completo}

Figura 4 - vista superior com medições das distâncias entre os pontos marcados e o eixo central, aferindo o comprimento das folhas

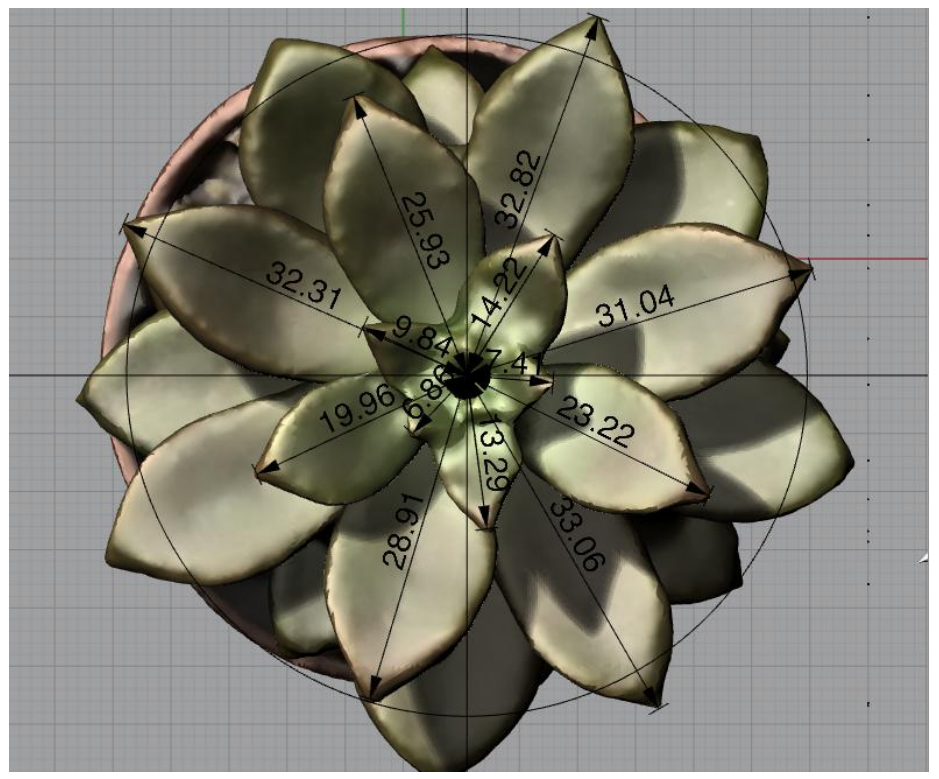

Fonte: os autores

Outra tarefa executada foi a medição da distância entre cada ponto extremo das folhas até a linha de base do vaso, o que permitiu a aferição da distância existente entre cada folha e a linha de base. Esses dados possibilitaram o início da reflexão sobre a existência de um padrão de crescimento vegetal no objeto de estudo. Os dados obtidos podem ser observados na Figura 5.

Figura 5 - Vistas laterais: (a) alturas do ponto extremo da folha até linha do vaso e (b) alturas do ponto extremo da folha até linha do vaso.
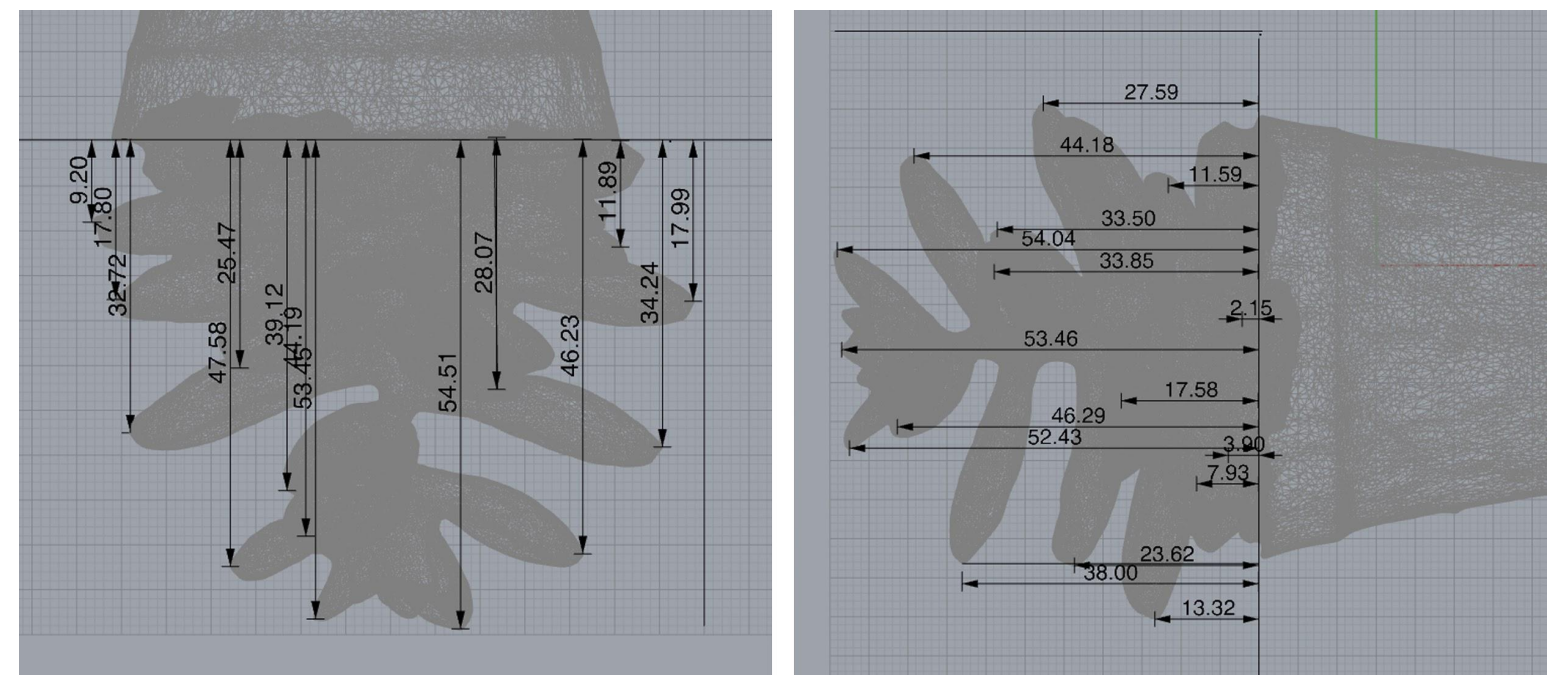

Fonte: os autores 


\section{Artigo Completo}

\section{Resultados e Discussão}

Por meio do estudo computacional do modelo 3D virtual, foi possível identificar alguns padrões geométricos, especialmente espirais de crescimento. Estas espirais foram então analisadas comparativamente à espiral de Fibonacci.

\subsection{Identificação dos Padrões Geométricos}

Passadas as aferições das primeiras medidas, seguiu-se no processo de identificação de geometrias. O primeiro padrão geométrico identificado foi a respeito da filotaxia, que se refere ao arranjo e disposição das folhas no caule. Segundo Haudin e Residori (2013), diferentes modos de crescimento podem ser identificados, dentre eles a disposição oposta Dística, oposta Cruzada, a Verticilada e a Espiralada. No caso do modelo em questão, identificou-se o padrão de filotaxia Espiralada, pois as folhas sucedem-se ao longo do caule em uma linha ascendente de percurso helicoidal. Na projeção em planta baixa, é possível perceber que os pontos desta espiral (coincidentes com as pontas das folhas) vão se sucedendo conformando triângulos de diferentes dimensões (Figura 6).

Figura 6 - união dos pontos gerando linha com padrão geométrico triangular.

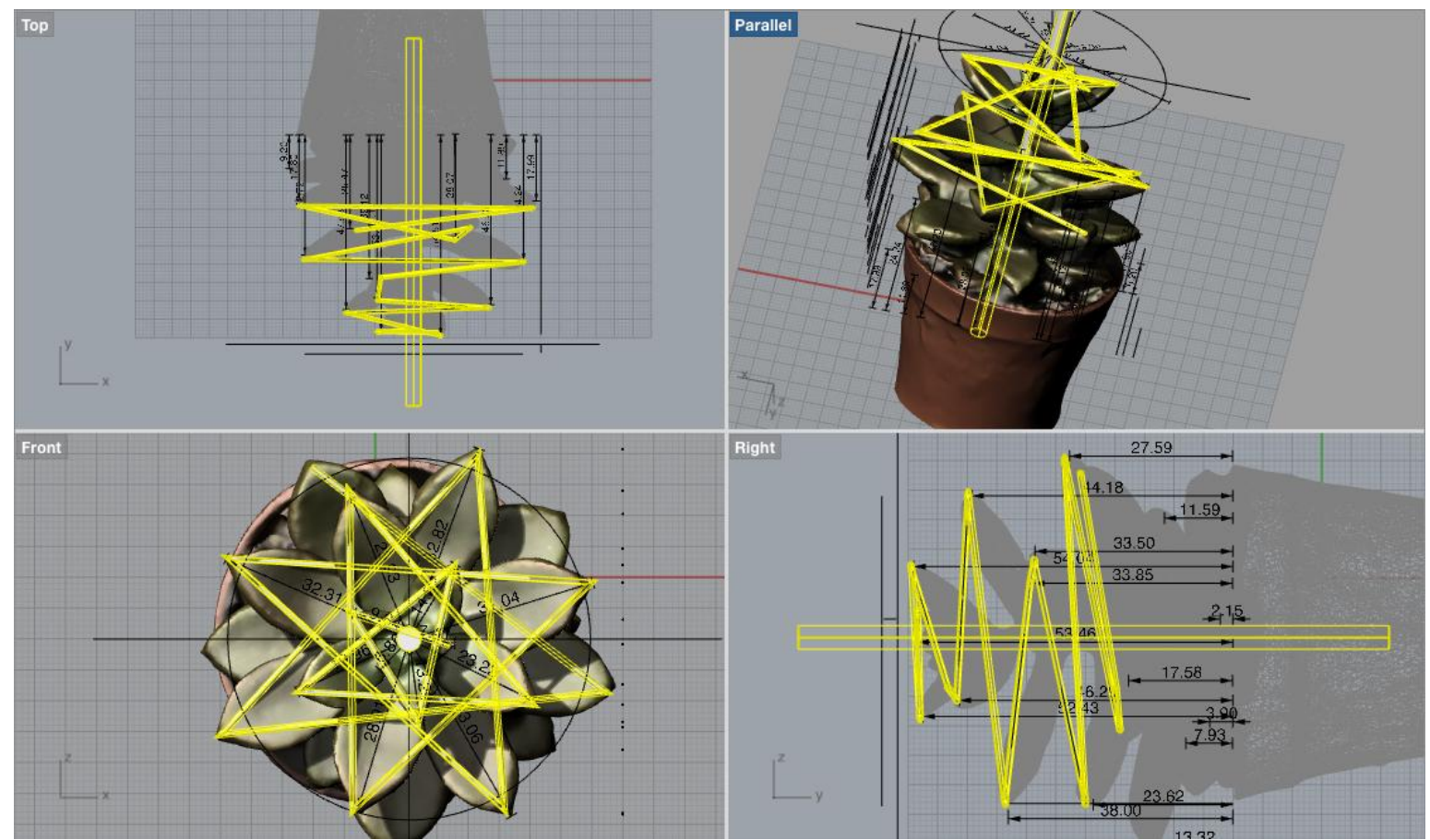

Fonte: os autores

Segundo Cruz (2012), espiral é uma das formas mais amplamente encontradas na natureza. Elas podem ser divididas em espirais constantes, como as teias de aranha, e algorítmicas, como as conchas Nautilus. Enquanto as espirais constantes têm um crescimento uniforme, as algorítmicas crescem segundo um somatório das partes anteriores, como o caso da sequência de Fibonacci. 
Uma observação mais atenta da disposição das folhas no caule permite identificar não apenas uma hélice, mas uma sequência de hélices como geratrizes do crescimento do vegetal. Seguindo etapas semelhantes às realizadas por Silva (2017) na construção das curvas geométricas da casca do Abacaxi, foi possível mapear algumas das curvas existentes no modelo da suculenta. Para traçar as curvas, inicialmente foram desenhadas polilinhas tomando como referências os vértices da extremidade das folhas (Figura 7).

Figura 7 - traçado das curvas de crescimento com polilinhas

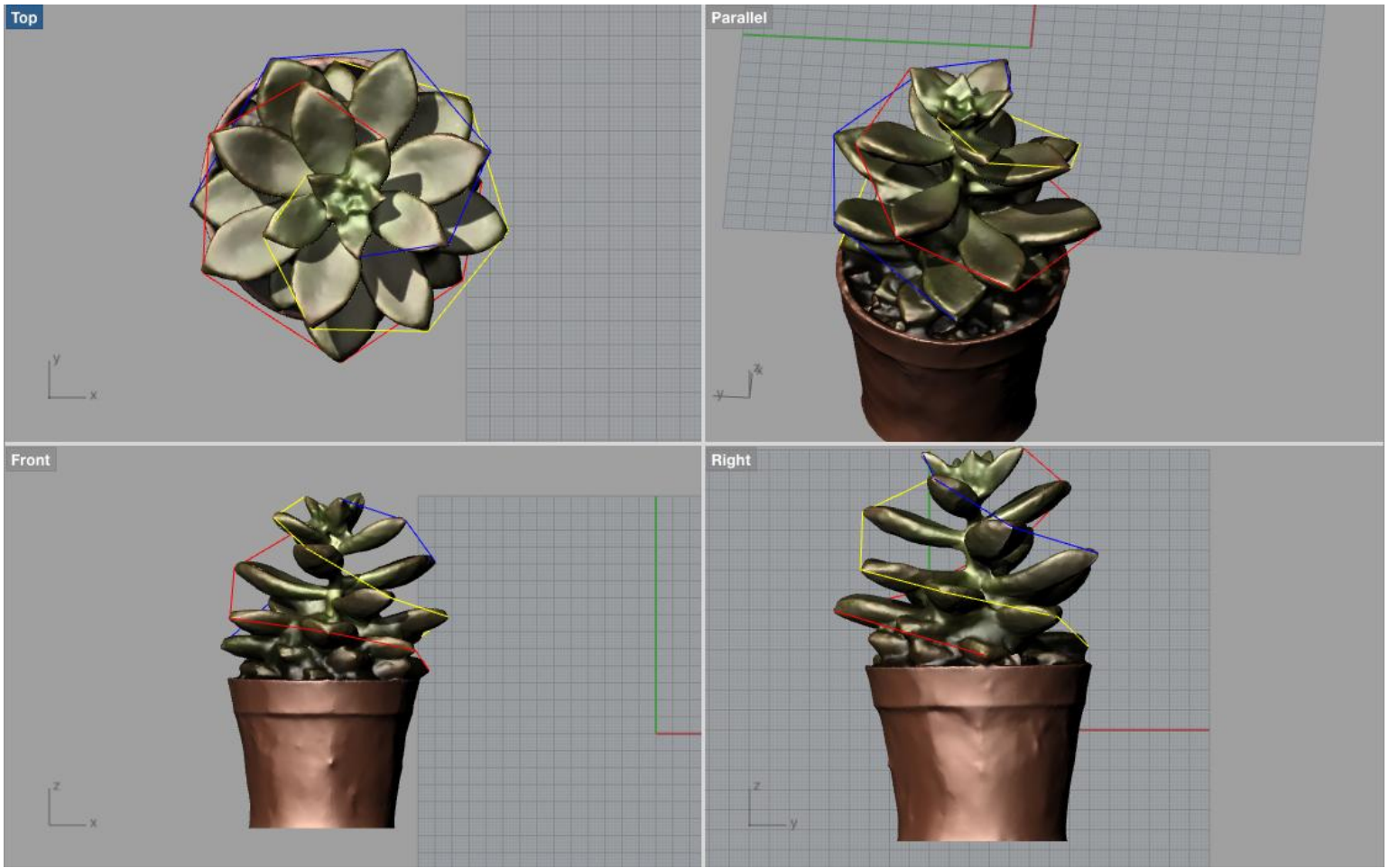

Fonte: os autores

Sobre as polilinhas foi possível construir as curvas com pontos interpolados, gerando, em planta, espirais e, tridimensionalmente, hélices. As três hélices encontradas (Figura 8) possuem proporções muito semelhantes entre si, apenas apresentando ângulos de giro diferentes. 


\section{Artigo Completo}

Figura 8 - transformação das polilinhas em curvas com pontos interpolados

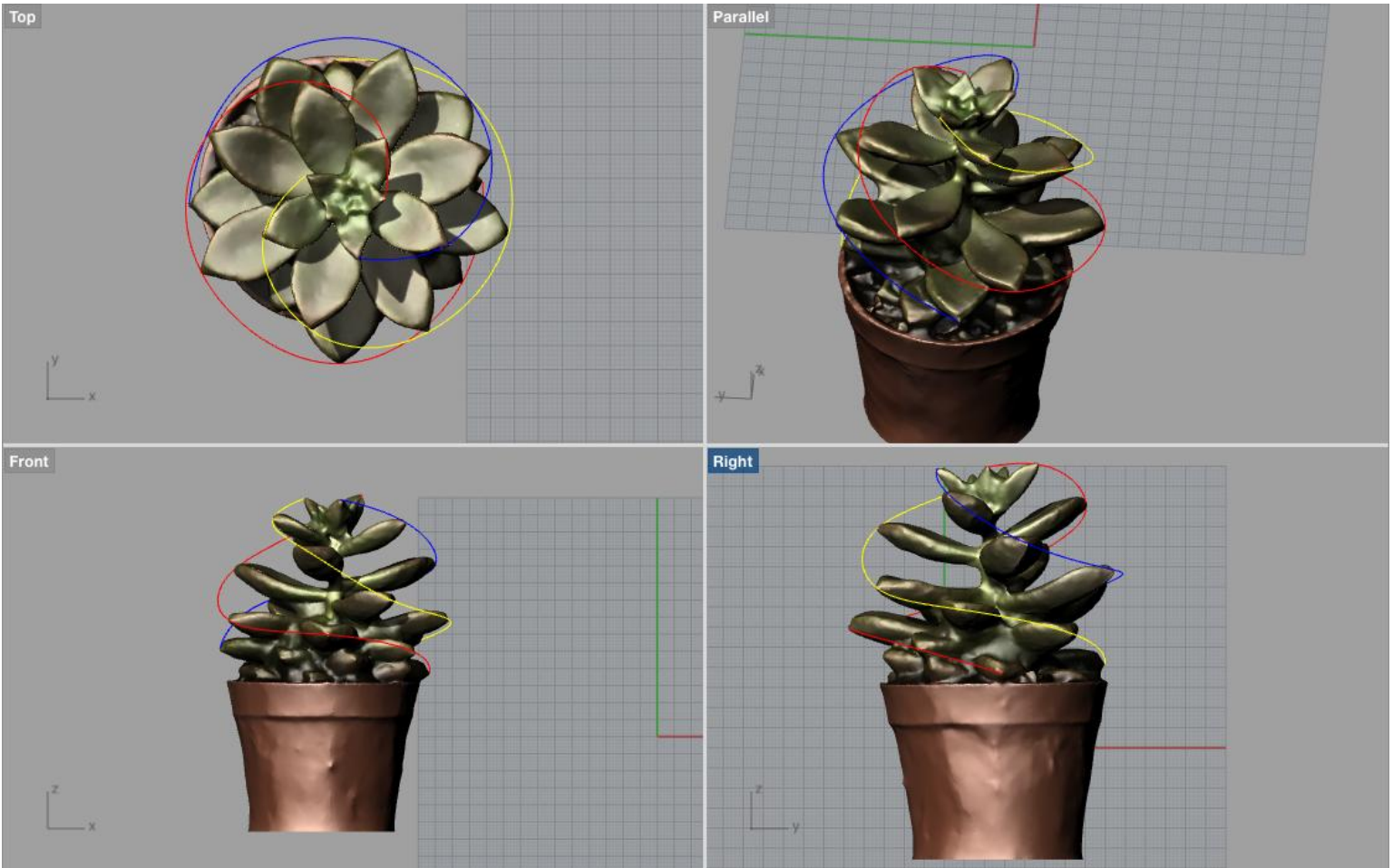

Fonte: os autores

\subsection{Identificação das semelhanças com Espiral de Fibonacci}

Visando o estabelecimento de similaridades entre a Espiral de Fibonacci e as geratrizes helicoidais encontradas no modelo 3D virtual obtido por meio de Fotogrametria Digital, foi construída no software Rhinoceros uma espiral com a sequência proposta por Fibonacci, a partir de uma malha quadriculada de $1 \times 1 \mathrm{~cm}$. Sobre essa malha foram desenhados os quadrados referentes à sequência de Fibonacci $(1,1,2,3,5,8$ e 13). A etapa seguinte foi o traçado de um arco inscrito em cada um dos quadrados ( $1 / 4$ de circunferência), gerando a espiral que está destacada em amarelo na Figura 9.

Figura 9 - construção da Espiral de Fibonacci.

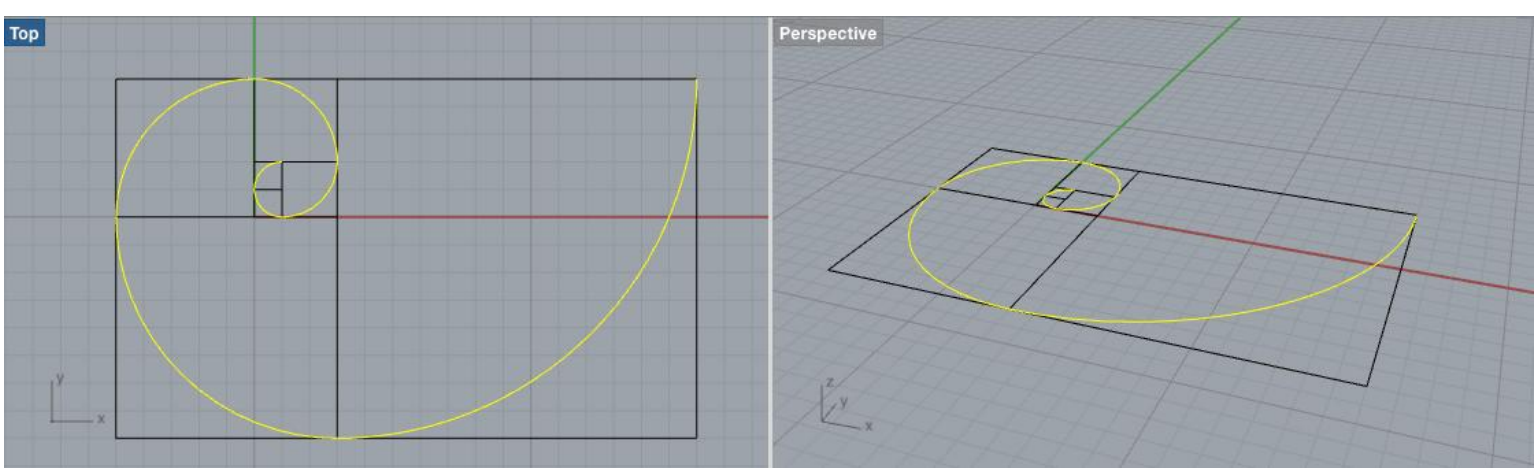

Fonte: os autores 


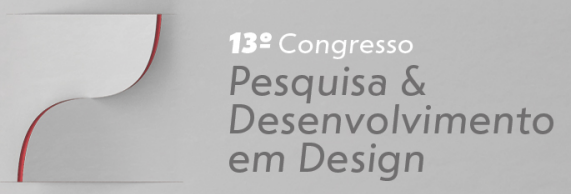

\section{Artigo Completo}

A espiral foi então inserida junto ao modelo da suculenta e suas geratrizes helicoidais. Quando projetadas na vista superior (planta baixa), as hélices transformadas em espirais, se assemelham às proporções de Fibonacci. A figura 10 ilustra cada uma das geratrizes encontradas no modelo da suculenta, rotacionadas para coincidirem uma sobre a outra, sendo comparadas diretamente com a espiral de Fibonacci (curva preta).

Figura 10 - Comparativo entre geratrizes helicoidais extraídas do modelo 3D e espiral de Fibonacci

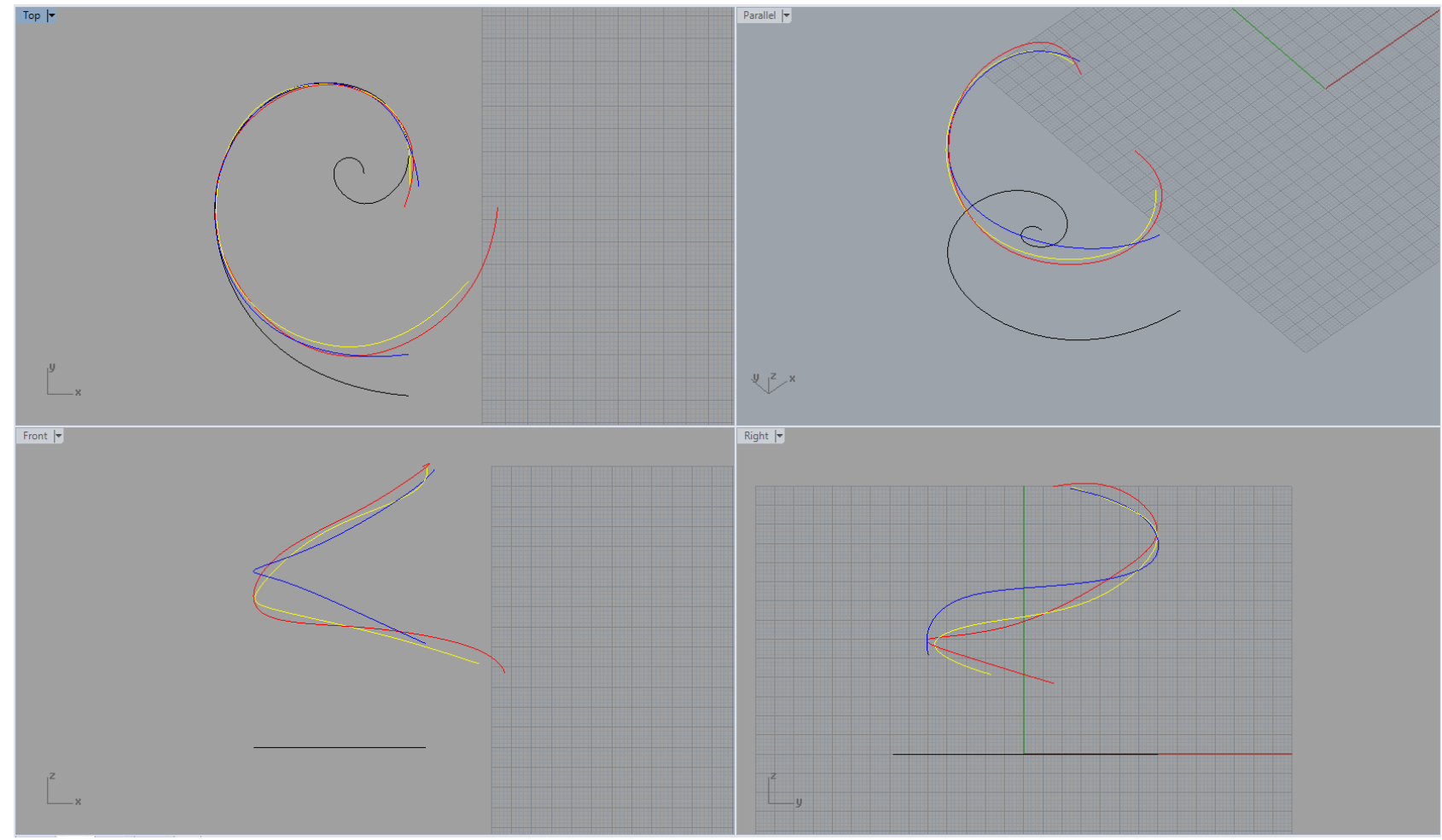

Fonte: os autores

A compatibilidade geométrica observada entre projeção das hélices das suculentas e a espiral de Fibonacci confirma outros estudos que demonstram este tipo de lei geradora regendo a configuração formal de seres vivos. Estre tipo de estudo abre caminho para o desenvolvimento de algoritmos e softwares generativos para a geração de geometrias de seres vivos simulados a partir das parametrizações realizadas. Assim, seria possível simular um grande número de indivíduos utilizando, por exemplo, algoritmos genéticos para prever um determinado comportamento natural. Outra possibilidade seria a utilização destas parametrizações como base para a geração de geometrias de produtos em função de um determinado tipo de desempenho que possa ser beneficiado pelo tipo de geometria analisada.

\section{Considerações Finais}

A parametrização de sistemas e organismos naturais tem trazido novas perspectivas para o design, oportunizando consistentes referências no que concerne a estruturas duradouras, resistentes e econômicas. A natureza, após 3,6 bilhões de anos de experiência, aprendeu a se aprimorar e o resultado é o que mais perdura. Analisar as suas estruturas é uma chave para a 
criatividade e a inovação.

A digitalização tridimensional da Flor Fantasma (nome científico: Graptopetalum paraguayense) possibilitou a geração de um modelo 3D para ser estudado virtualmente. O modelo foi submetido a análise e manipulação digital, o que possibilitou identificar uma geometria com geratrizes helicoidais similares às encontradas na Espiral de Fibonacci. Diferentemente da natureza de informações que podem ser extraídas de modelos bidimensionais e fotos, o modelo em 3D possibilitou um campo mais amplo de exploração, com a capacidade de identificação de geometrias mais complexas, extrapolando as limitações da linguagem bidimensional.

Os resultados apresentados reforçam a informação de que a natureza se organiza e se desenvolve a partir de padrões matemáticos específicos, não aleatoriamente. As imagens demonstram que a Espiral de Fibonacci, de alguma forma, regula o crescimento do vegetal, como uma geratriz a partir da qual se distribuem organizadamente todos os seus componentes. As parametrizações de geometrias da natureza pode dar origem a algoritmos de simulação e também para o desenvolvimento de geometrias de produtos baseadas na natureza de forma a melhorar o seu desempenho.

A partir do modelo virtual gerado podem ainda ser aferidas outras dimensões, para seguir avançando em detalhamento e análises. Um estudo mais detalhado sugerido poderia incluir as medidas de desvio de cada uma das curvas do modelo com relação à referência de Fibonacci, de forma a identificar o seu grau de imprecisão. Ainda poderiam ser traçados paralelos com curvas de outras estruturas vegetais como o abacaxi, a pinha e até mesmo de outras espécies de suculentas.

O trabalho desenvolvido ratifica a presença de um princípio de solução da natureza, neste caso geométrico, para futuras aplicações junto à geração de novos produtos. Partindo do pressuposto que a natureza possui soluções de extrema eficácia, reproduzir seus princípios no design de produtos significa aprimorá-los em âmbito funcional, estrutural, econômico e estético. Para tangibilizar essa aplicação, as curvas identificadas no modelo da suculenta poderiam ser parametrizadas de maneira a estabelecer um critério de movimento/crescimento/expansão a ser tomado como referência para inúmeros usos, incluindo a possibilidade de desenvolvimento de ferramenta computacional para aplicações biomiméticas.

\section{Referências}

ARÉVALO VERA, B. et al. Metodología para documentación 3D utilizando fotogrametría digital. Tecnura, dez. 2015. v. 19, n. spe, p. 113-120.

CARO, J. L. Fotogrametría y modelado 3D: un caso práctico para la difusión del patrimonio y su promoción turística. In: IX CONGRESO TECNOLOGÍAS DE LA INFORMACIÓN Y LAS COMUNICACIONES, 2012, España. Anais. Universidad de Málaga, 2012. p. 1-15.

CRUZ, A. J. A. B. Arquitectura biológica: uma análise da obra de Frei Otto. Coimbra: Faculdade de Ciências e Tecnologia da Universidade de Coimbra, 2012. Dissertação de Mestrado.

DANTAS, P. V. De F. et al. Protótipo de dispositivo facilitador para digitalização 3D por fotogrametria com smartphones. In: XX CONGRESO DE LA SOCIEDAD IBEROAMERICANA DE GRÁFICA DIGITAL, 2016, [s.I.]. Anais eletrônicos. [S.I.]: [s.n.], 2016. V. 3, p. 297-304. Disponível em: $<$ http://www.proceedings.blucher.com.br/article-details/prottipo-de-dispositivo-facilitador-para- 
digitalizao-3d-por-fotogrametria-com-smartphones-24809>. Acesso em: 4 ago. 2017.

DOCZI, G. O Poder dos Limites: harmonias e proporções na natureza, arte e arquitetura. São Paulo: Mercuryo, 1990.

FOSTER, S.; HALBSTEIN, D. Integrating 3D Modeling, Photogrammetry and Design. London: Springer London, 2014.

HAUDIN, F.; RESIDORI, S. Golden spirals as phyllotactic arrangements of optical patterns. Physical Review E, 8 fev. 2013. v. 87, n. 2, p. 020901.

HEIPKE, C. State-of-the-Art of Digital Photogrammetric Workstations for Topographic Applications. Photogrammetric Engineering \& Remote Sensing, jan. 1995. v. 61, n. 01, p. 49-56.

HSUAN-AN, T. Sementes do Cerrado e Design Contemporâneo. Goiânia: Universidade Católica de Goiás, 2002.

LINDER, W. Digital Photogrammetry. 3. ed. Berlin, Heidelberg: Springer Berlin Heidelberg, 2009.

PHOTOGRAMMETRY. In: Britannica Digital Learning. [S.I.]: Encyclopædia Britannica, Inc., 2017. Disponível em: <http://academic-ebbritannica.ez45.periodicos.capes.gov.br/levels/collegiate/article/photogrammetry/59808>. Acesso em: 4 ago. 2017.

SCHINDLER, K.; FÖRSTNER, W. Photogrammetry. ETH Zurich. Disponível em: <https://www.ethz.ch/content/dam/ethz/special-interest/baug/igp/photogrammetry-remotesensing-dam/documents/pdf/photogrammetry.pdf>. Acesso em: 3 jul. 2017.

SILVA, D. C. Da. Evolução da Fotogrametria no Brasil. Revista Brasileira de Geomática. Universidade Tecnológica Federal do Paraná, Pato Branco, PR, Brasil, dez. 2015. v. 3, n. 2, p. 8196.

SILVA, L. S. Da. Design Paramétrico a partir da Digitalização 3D de Geometrias da Natureza com Padrão de Crescimento Espiral. Brasil: Universidade Federal do Rio Grande do Sul, 2017. Dissertação de Mestrado.

Sketchfab. How to set up a successful photogrammetry project. Sketchfab Blog, [S.I.], 18 jun. 2015. Disponível em: <https://goo.gl/SZCf3g>. Acesso em: 31 maio 2017.

TEMBA, P. Fundamentos da Fotogrametria. Departamento de Cartografia - UFMG. Disponível em: <http://csr.ufmg.br/geoprocessamento/publicacoes/fotogrametria.pdf>. Acesso em: $1^{\circ}$ ago. 2017. paraguayense) possibilitou a geração de um modelo 